First publ. in: Physical Review / A 37 (1988), 7, pp. 2716-2718

\title{
Photodetachment spectroscopy of cold aluminum cluster anions
}

\author{
G. Ganteför, K. H. Meiwes-Broer, and H. O. Lutz \\ Fakultät für Physik, Universität Bielefeld, D-4800 Bielefeld 1, Federal Republic of Germany
}

\begin{abstract}
Aluminum cluster anions are produced in a laser vaporization source, cooled by adiabatic expansion into high vacuum, and mass-selected in a time-of-flight spectrometer. Pulsed uv light is used for electron detachment; the photoelectrons undergo energy analysis in a magneticbottle-type spectrometer. The resulting electron spectra reflect the band-structure development of aluminum clusters.
\end{abstract}

As with molecules and surfaces, photoelectron spectroscopy is expected to provide important information on the electronic structure of atomic clusters. To unambiguously interpret such experimental information, clusters should be in a well-characterized state. Most importantly, they must be mass selected; so far, there exists no reliable method to mass select neutral clusters. Furthermore, the parent clusters should be cold to reduce the ambiguities associated with ill-defined vibrational excitations. Therefore we decided to investigate the energy of photodetached electrons of cold negatively charged clusters with defined mass. Fragmentation after detachment (low photon fluxes assumed) does not influence the outcome of the experiment as the photoelectrons stem from the ion's ground state.

The experimental procedure has been described elsewhere. $^{1,2}$ In short, a laser vaporization source similar to the standard technique is used, i.e., a target rod of the desired metal is mounted in a pulsed, high-pressure supersonic nozzle. ${ }^{3,4}$ Light pulses from an excimer laser vaporize the metal, the resulting plasma being flushed out by a pulse of $\approx 1$ atm helium or neon through a channel of variable length into high vacuum. We do not use a postionizer; instead, we study the charged clusters as they emerge from the source. In order to avoid any disturbance of the supersonic expansion, the area between nozzle and skimmer is carefully shielded from electric fields. From the expanding beam, ion bunches are formed by appropriate potential switching; they are space- and timefocused collinearly to the source direction into the electron spectrometer. The $1-\mathrm{m}$ ion drift tube is sufficiently long to separate the ion bunches as is demonstrated by the $\mathrm{Al}_{n}$ - mass spectrum (Fig. 1).

The envelopes of such intensity distributions can be changed by adjusting experimental parameters, but fine structures are weak or do not appear at all when the expansion is optimized to large clusters. "Hot" metal clusters, on the other hand, which have been heated, e.g., during the production by sputtering, 1,5 show marked intensity steps as the result of unimolecular decomposition of the excited ions; in the case of $\mathrm{Al}_{n}$ - a huge intensity step occurs after $n=13$. Such a structure is completely miss-

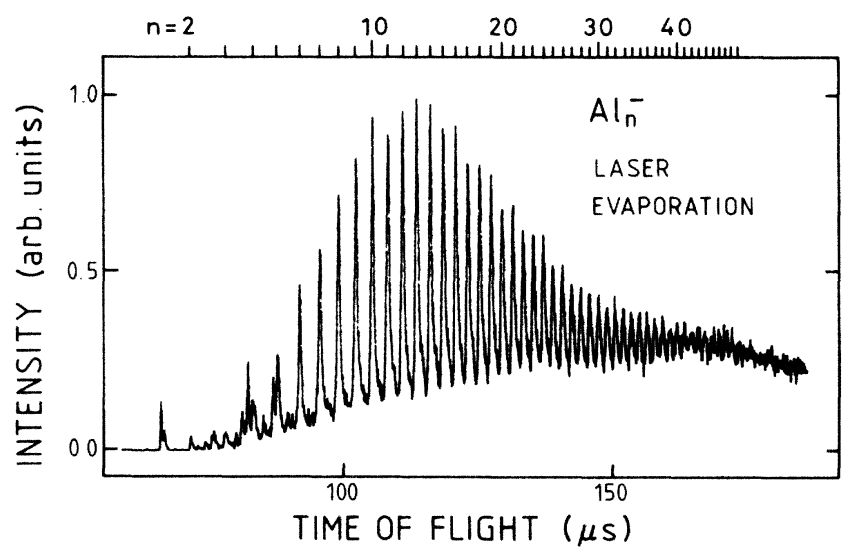

FIG. 1. Time-of-flight mass spectrum of $\mathrm{Al}_{n}{ }^{-}$obtained out of the laser vaporization source, without postionization. The ion bunches have an energy of $250 \mathrm{eV}$ and are formed by $50-\mathrm{V}$ grid switching. 


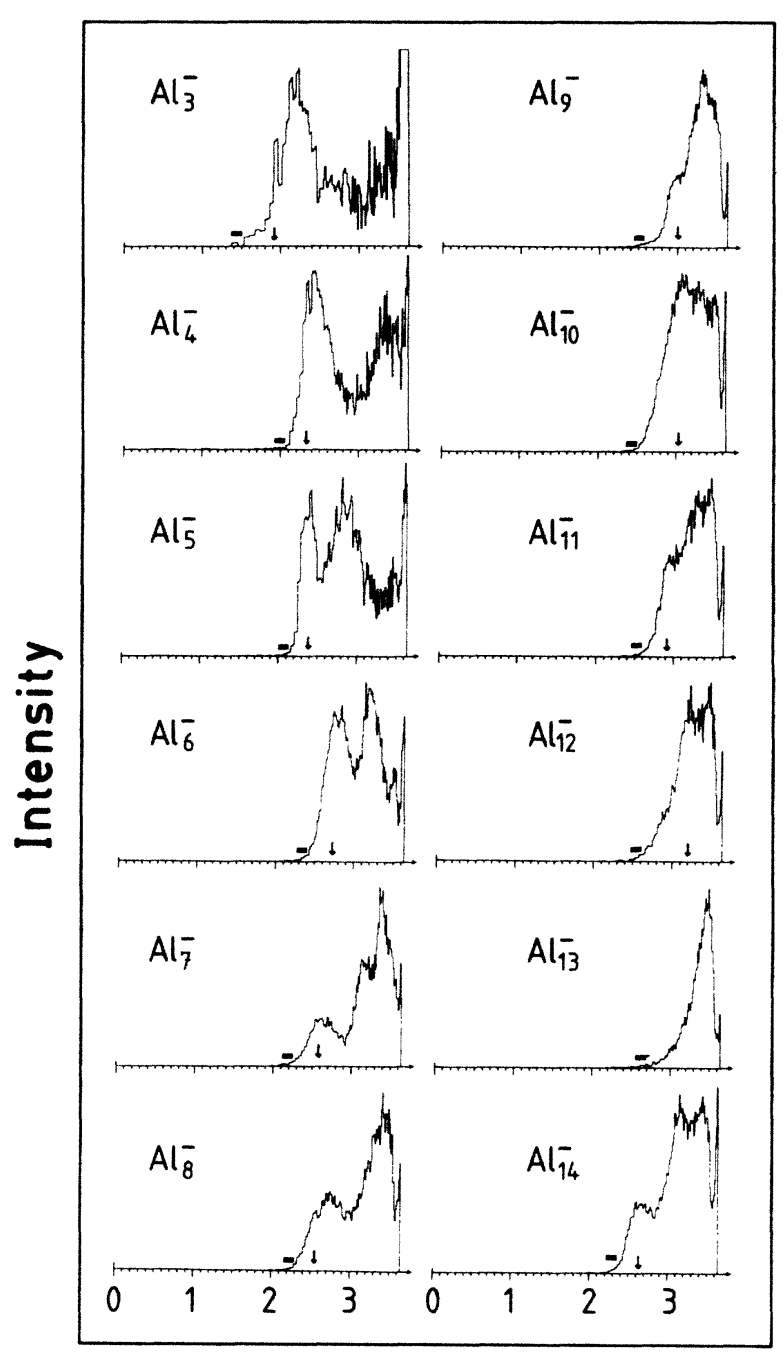

\section{Electron binding energy $(\mathrm{eV})$}

FIG. 2. Photoelectron spectra of $\mathrm{Al}_{n}$ - clusters $(n=3-14)$ at a detachment energy of $3.68 \mathrm{eV}(337 \mathrm{~nm})$. Each spectrum is an average over $6.4 \times 10^{4}$ shots. The background signal is negligible on the scale shown and has therefore not been corrected for. The high-energy maxima beyond $3.5 \mathrm{eV}$ are an artifact of the experimental method.

ing in Fig. 1, thus proving that the temperature of $\mathrm{Al}_{n}{ }^{-}$, obtained directly from the laser vaporization source, is at least below the boiling point. Additionally, a line-shape analysis yields translational temperatures of about $10 \mathrm{~K}$.

For photoelectron spectroscopy of cluster ions, a second uv laser $\left(\mathrm{N}_{2}, \hbar \omega=3.68 \mathrm{eV}\right)$ is fired into the detachment area which is designed to be the starting region of a magnetic-bottle time-of-flight spectrometer. ${ }^{2,6}$ The firing time of the second laser is tuned to be in coincidence with the arrival of a defined ion bunch. Without further acceleration the electrons start in a strongly divergent magnetic field and are adiabatically guided into the weak-field drift tube of $0.65-\mathrm{m}$ length. The overall resolution of this setup is $\Delta E_{e} / E_{e}=0.06$. The resulting electron time-offlight (TOF) spectra are converted into energy spectra shown in Fig. 2. The energy scale is calibrated by recording known photoelectron spectra of $\mathrm{Cu}^{-}, \mathrm{Cu}_{n}{ }^{-}$, and $\mathrm{Fe}_{2}-2,7,8$

As a first direct result the spectra provide photodetachment onset energies (marked by bars in Fig. 2). These thresholds are estimates of vertical electron affinities (EA) within an uncertainty of approximately $\pm 0.2 \mathrm{eV}$ and are not thought to be origin transitions. The degree of geometrical change of $\mathrm{Al}_{n}$ - upon neutralization is not known, i.e., no information is yet available about the difference between vertical and adiabatic electron affinities. Therefore, the onsets give upper limits of the adiabatic electron affinities. The observed onset energies of $\mathrm{Al}_{n}{ }^{-}$(compiled in Table I) show a strong increase for low $n$, followed by a weak rise for larger $n$. A qualitatively similar behavior of the EA has been calculated by Upton for $n \leq 6 .^{9}$

On the assumption that the initial state is well defined (i.e., there exist no ionic isomers) the measured photoelectron bands reflect ground and excited states of the neutral clusters. A special feature of the spectra is the occurrence of a fairly separated low-energy maximum for nearly all $n$, the only exception being $\mathrm{Al}_{13}{ }^{-}$. The corresponding energies $E_{\max }$ are marked by arrows in Fig. 2 . These peaks (note that their width is much larger than the instrumental resolution) do not necessarily stem only from the ground state. For aluminum different low-lying neutral excited states resulting from a set of spin multiplicities have been calculated to lie within approximately $1 \mathrm{eV}$ above the ground state. ${ }^{9,10}$ The fine structure within the first maxima might hint to such narrow-spaced states. Most of the spectra show additionally one or two broad high-energy peaks which could correspond to higher neutral states. The widths and the positions of these features vary irregularly with $n$, possibly indicating the band development of aluminum clusters.

Of all systems presented in Fig. 2 the first band of $\mathrm{Al}_{13}{ }^{-}$lies at an extraordinarily high energy. This behavior shows resemblance to predictions of the electron droplet model in which $\mathrm{Al}_{13}$ - is supposed to be a closed-shell cluster. ${ }^{11}$ Therefore, all valence electrons are needed to fill the shell, i.e., the electron binding energy should be high.

TABLE I. Photoelectron threshold energies $\left(E_{\mathrm{th}}\right)$ and maximum positions $\left(E_{\max }\right)$ of the low-energy bands for $\mathrm{Al}_{n}^{-}, n=3 \ldots 14$ (in eV).

\begin{tabular}{ccccccccccccc}
\hline$n$ & 3 & 4 & 5 & 6 & 7 & 8 & 9 & 10 & 11 & 12 & 13 & 14 \\
\hline$E_{\text {th }}$ & 1.4 & 2.0 & 2.1 & 2.3 & 2.2 & 2.2 & 2.5 & 2.4 & 2.5 & 2.6 & 2.6 & 2.3 \\
$E_{\max }$ & 1.9 & 2.3 & 2.4 & 2.7 & 2.6 & 2.6 & 3.0 & 3.1 & 2.9 & 3.2 & $>3.5$ & 2.6 \\
\hline \hline
\end{tabular}


During the course of these experiments, Leopold, Ho, and Lineberger, ${ }^{7}$ Chesnovsky et al. , ${ }^{12}$ and Bowen et al. ${ }^{13}$ also investigated photoelectrons of metal cluster anions. It can be expected that the powerful technique of ultraviolet photoelectron spectroscopy (UPS) will be the key to the understanding of the electronic band structure as it evolves from atoms and molecules to the infinite condensed state.

${ }^{1}$ W. Begemann, S. Dreihöfer, G. Ganteför, H. R. Siekmann, K. H. Meiwes-Broer, and H. O. Lutz, in Elemental and Molecular Clusters, edited by T. P. Martin, Springer Series on Material Science, Vol. 6 (Springer, Berlin, 1988).

${ }^{2}$ G. Ganteför, M. Gausa, K. H. Meiwes-Broer, and H. O. Lutz, Z. Phys. D (to be published).

${ }^{3}$ T. G. Dietz, M. A. Duncan, D. E. Powers, and R. E. Smalley, J. Chem. Phys. 74, 6511 (1981).

${ }^{4}$ V. E. Bondybey and J. H. English, J. Chem. Phys. 74, 6978 (1981).

${ }^{5}$ W. Begemann, S. Dreihöfer, K. H. Meiwes-Broer, and H. O. Lutz, Z. Phys. D 3, 183 (1986).

${ }^{6}$ P. Kruit and F. H. Read, J. Phys. E 16, 313 (1983).
${ }^{7}$ D. G. Leopold, J. Ho, and W. C. Lineberger, J. Chem. Phys. 86, 1715 (1987).

${ }^{8}$ D. G. Leopold and W. C. Lineberger, J. Chem. Phys. 85, 51 (1986).

${ }^{9}$ T. H. Upton, J. Chem. Phys. 86, 7054 (1987).

${ }^{10}$ K. K. Sunil and K. D. Jordan (unpublished).

${ }^{11}$ M. Chou and M. Cohen, Phys. Lett. 113A, 410 (1986).

${ }^{12}$ O. Chesnovsky, P. J. Brucat, S. Yang, C. L. Pettiette, J. J. Craycraft, and R. E. Smalley, in The Physics and Chemistry of Small Clusters, edited by P. Jena, S. Khanna, and B. Rao, NATO Advanced Study Institute, Series B, Physics, Vol. 158 (Plenum, New York, 1987).

${ }^{13} \mathrm{~K}$. H. Bowen (unpublished). 